\title{
FTIR Nanospectroscopy Shows Molecular Structures of Plant Biominerals and Cell Walls
}

\section{Supporting Information}

Victor M. R. Zancajo, ${ }^{1,2,3}$ Tom Lindtner, ${ }^{1,2}$ Max Eisele, ${ }^{4}$ Andreas. J. Huber, ${ }^{4}$ Rivka Elbaum, ${ }^{1,5}$ Janina Kneipp ${ }^{1,2^{*}}$

${ }^{1}$ School of Analytical Sciences Adlershof (SALSA), Humboldt-Universität zu Berlin, 12489 Berlin, Germany

${ }^{2}$ Chemistry Department, Humboldt-Universität zu Berlin, 12489 Berlin, Germany

${ }^{3}$ BAM Federal Institute for Materials Research and Testing, 12489 Berlin, Germany

${ }^{4}$ Neaspec GmbH, D-85540 Munich-Haar, Germany

${ }^{5}$ The Robert H. Smith Institute of Plant Sciences and 563 Genetics in Agriculture, The Hebrew University of Jerusalem, 564 Rehovot 7610001, Israel

\section{Table of Contents}

Page

Figure S1. Spectra from microwave extracted phytoliths S2

Figure S2. Silica structures in phytoliths of sorghum leaves S3

Figure S3. Spectra of epoxy resin S4

Figure S4. SNOM image and spectra of silicified sclerenchyma S5

Figure S5. Mechanical phase image and profile of the sclerenchyma S5

Figure S6. Nano-IR spectra of fresh leaf sclerenchyma S6 


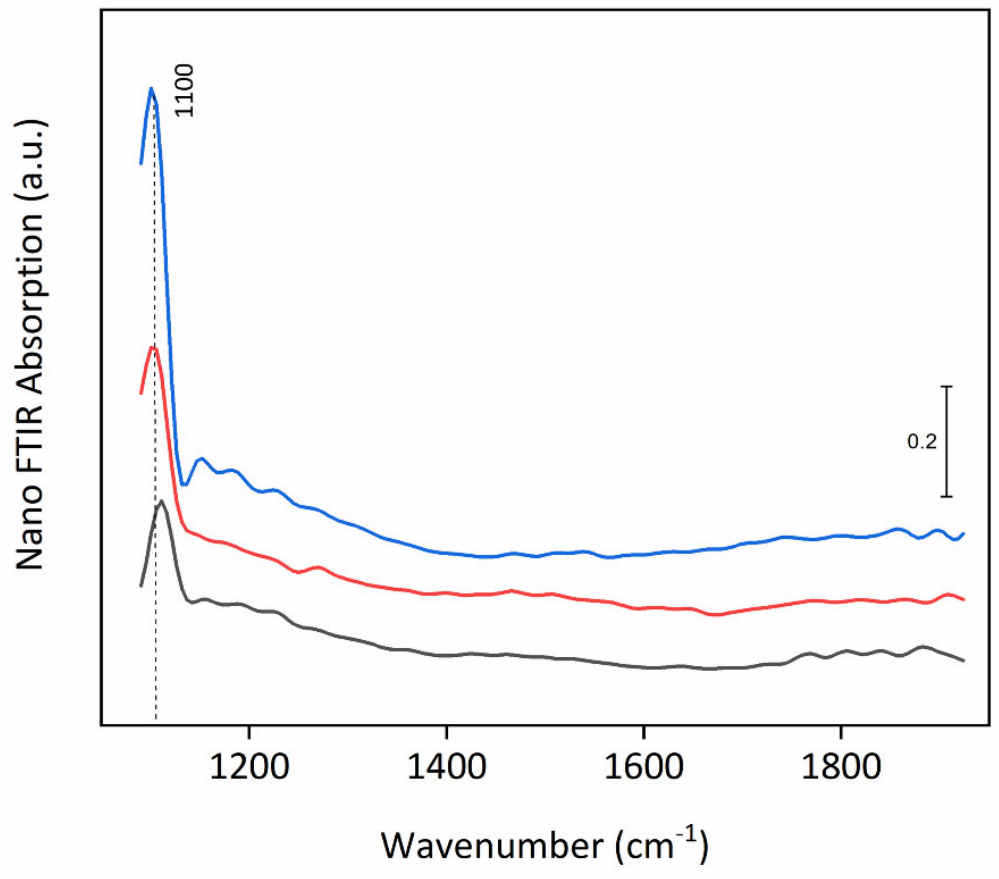

Figure S1. Spectra from microwave extracted phytoliths from different points of the same phytolith showing the strong silica phonon resonance. Spectra are off-set for clarity. 
A

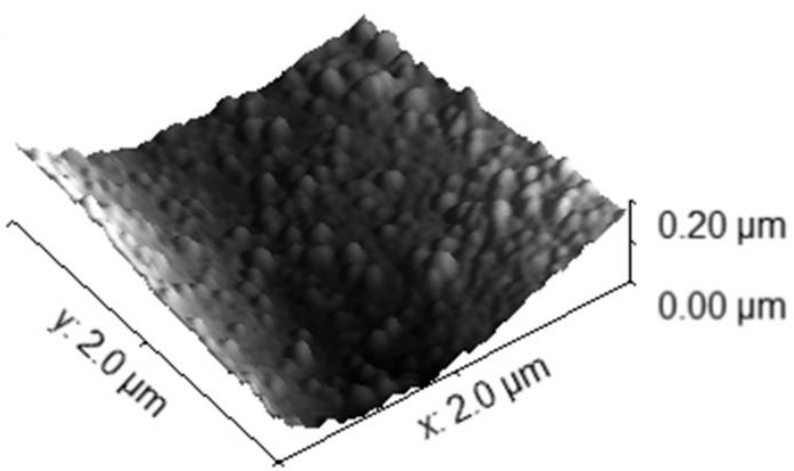

C

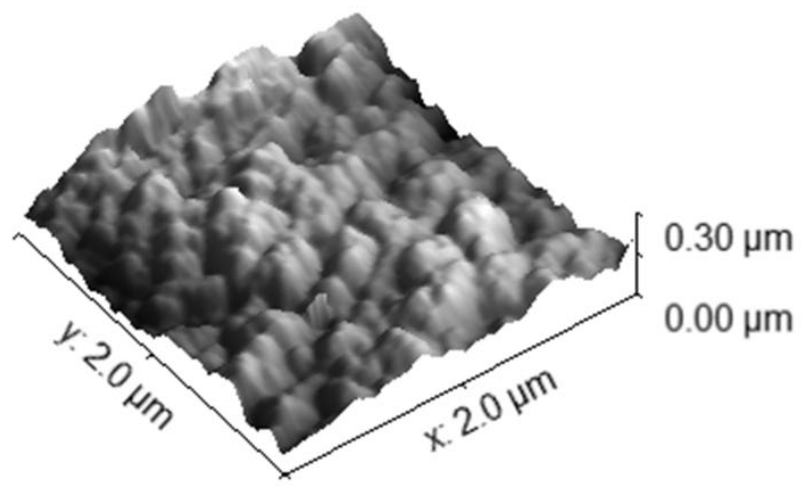

$\mathbf{E}$

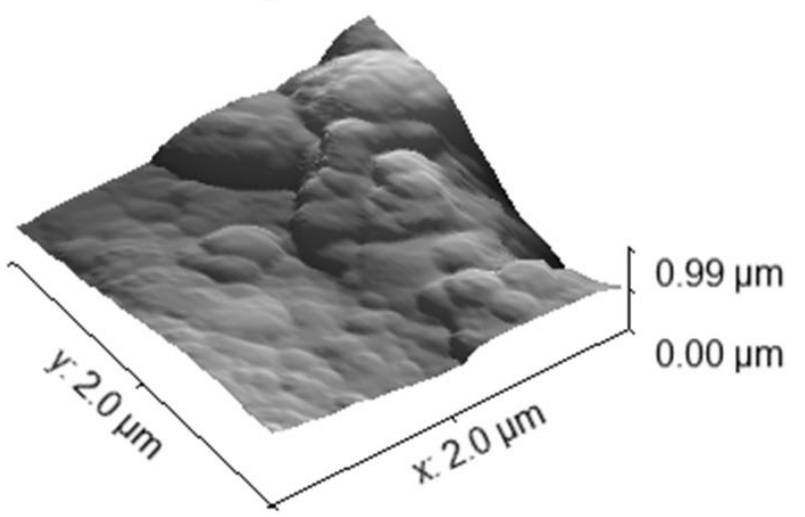

B

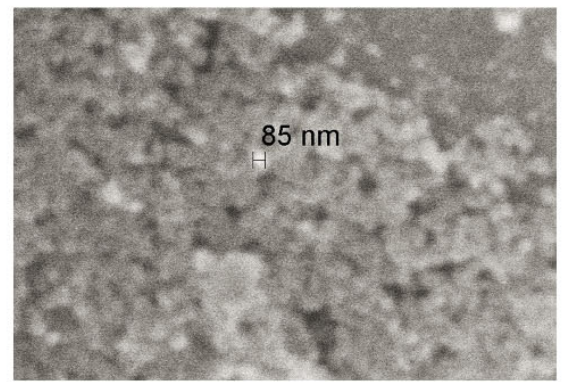

D

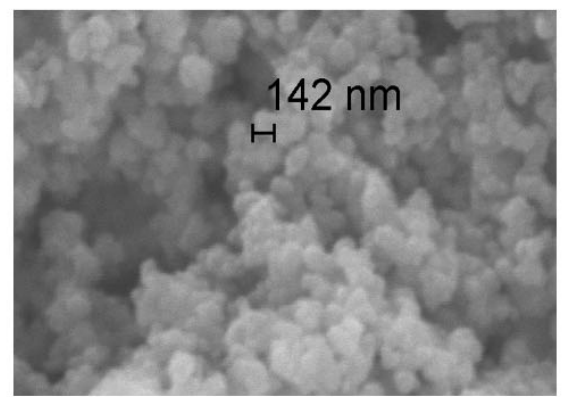

$\mathbf{F}$

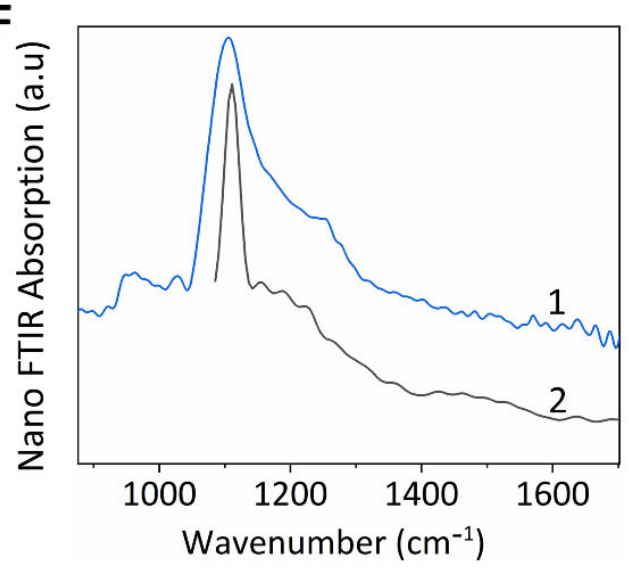

Figure S2. Silica structures in phytoliths of sorghum leaves. A) Three-dimensional (3D) topographic images of silica extracted by microwave assisted digestion showing small spherical particles. B) Scanning electron microscope (SEM) micrograph of extracted silica by microwave assisted digestion. C) 3D topographic images of silica extracted by wet digestion showing slightly bigger particles. D) SEM micrograph of extracted silica by wet digestion. E) Unextracted silica detected in situ in a leaf cross section showing a "cloudy" irregular structure. F) Nano-FTIR absorption spectra taken from silica phytoliths extracted by wet digestion (1) and microwave assisted digestion (2). 


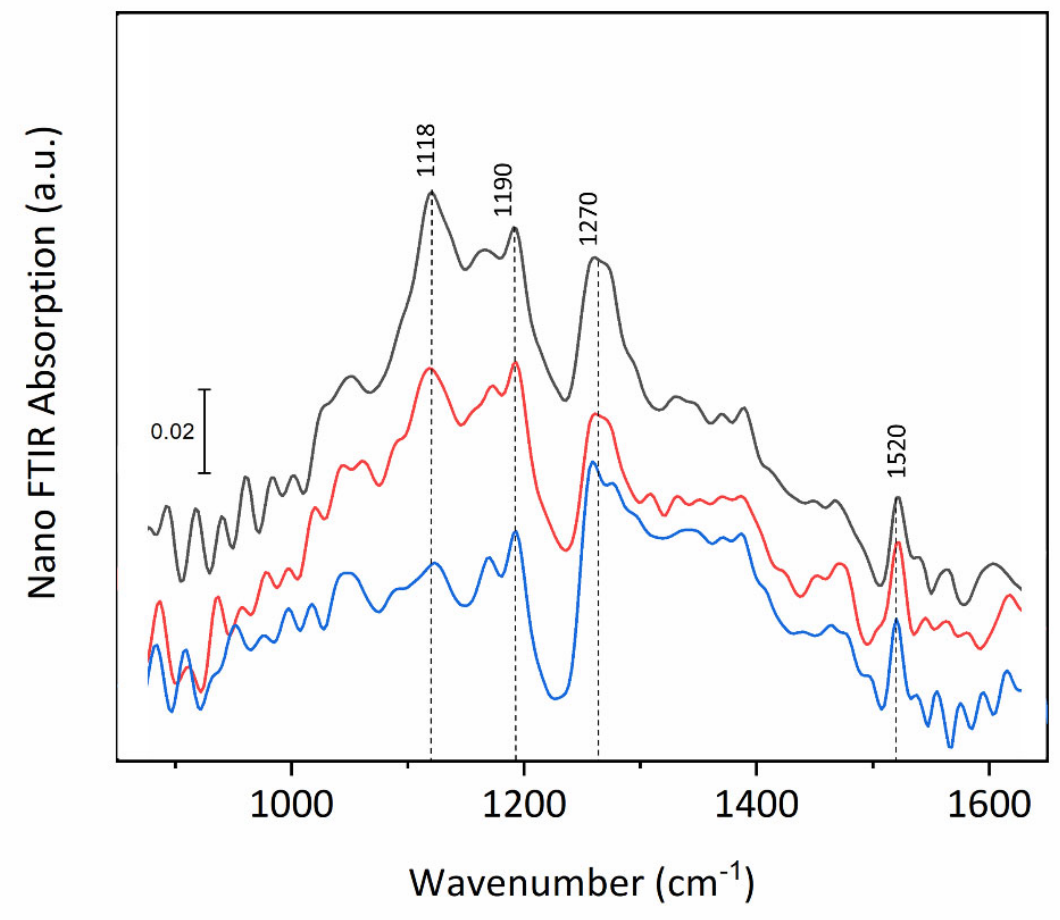

Figure S3. Spectra recorded at random spots from the epoxy resin (outside sample) used to embed the samples also are consistent in the band position this demonstrates reproducibility also for weak oscillators such organic compounds. Spectra are off-set for clarity. 
A

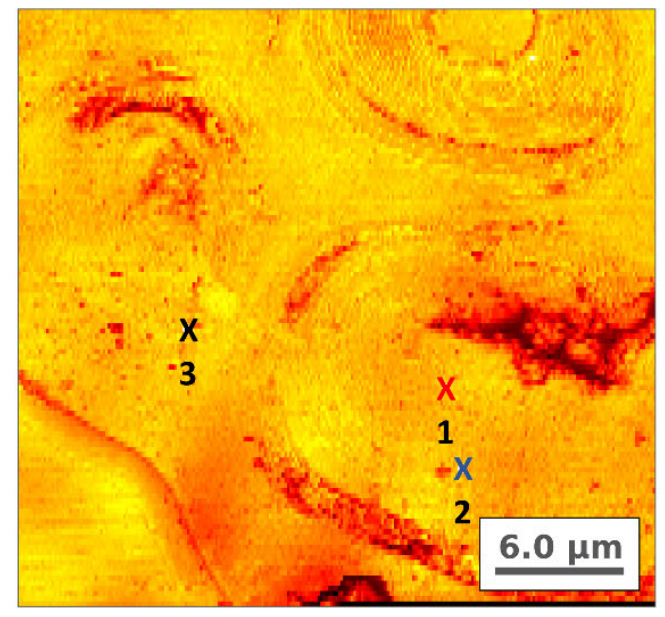

B

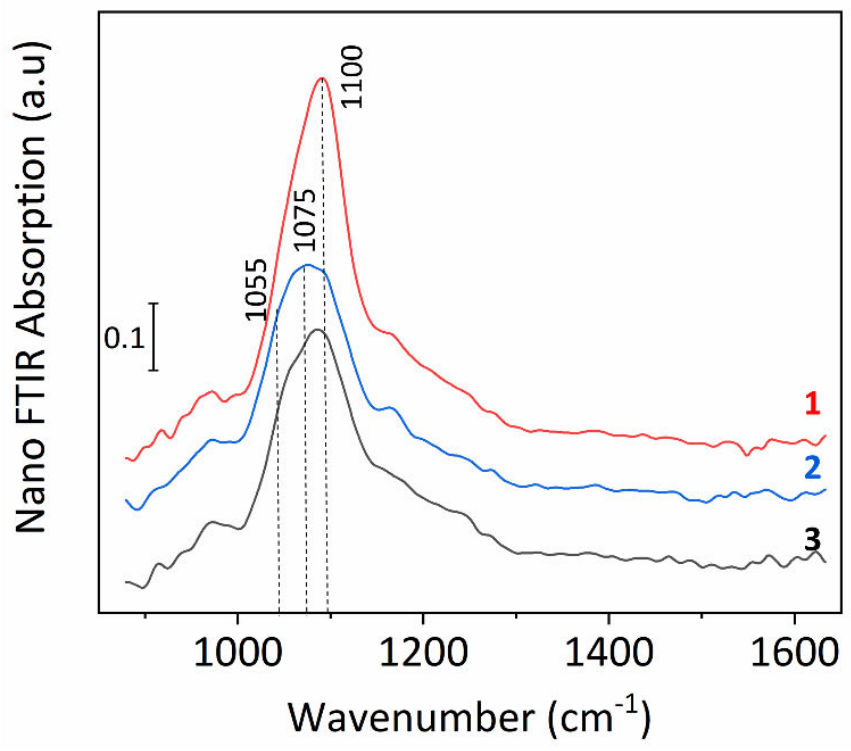

Figure S4. (A) Broadband spectrally integrated infrared SNOM image of silicified sclerenchyma, positions marked correspond to the shown in B. (B) Nano-FTIR absorption spectra showing the silica phonon resonance.

A

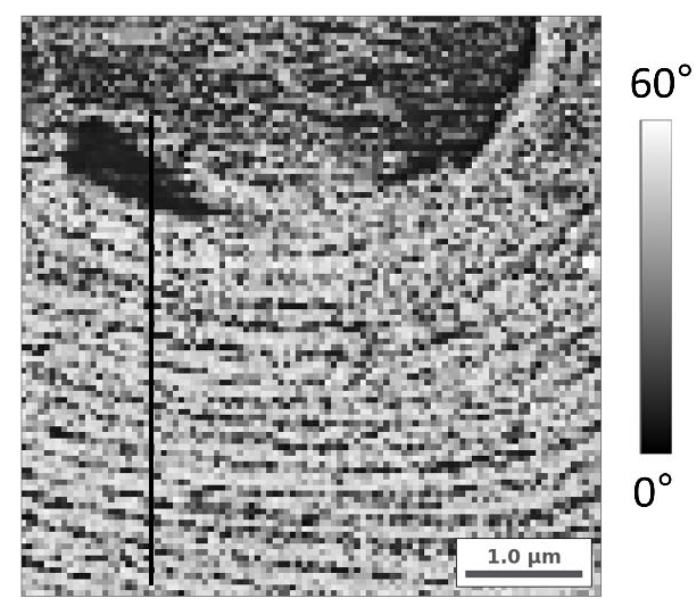

B

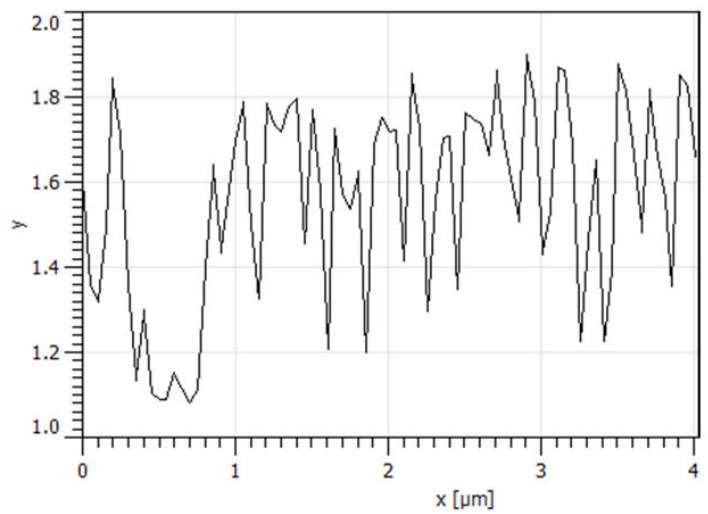

Figure S5. (A) Mechanical phase image of the sclerenchyma sample shown in Figure 3C. (B) mechanical phase profile of the line marked in (A), showing differences of $\sim 0.6$ rad between cell wall layers, the thickness of the layers is approximately $100 \mathrm{~nm}$. 

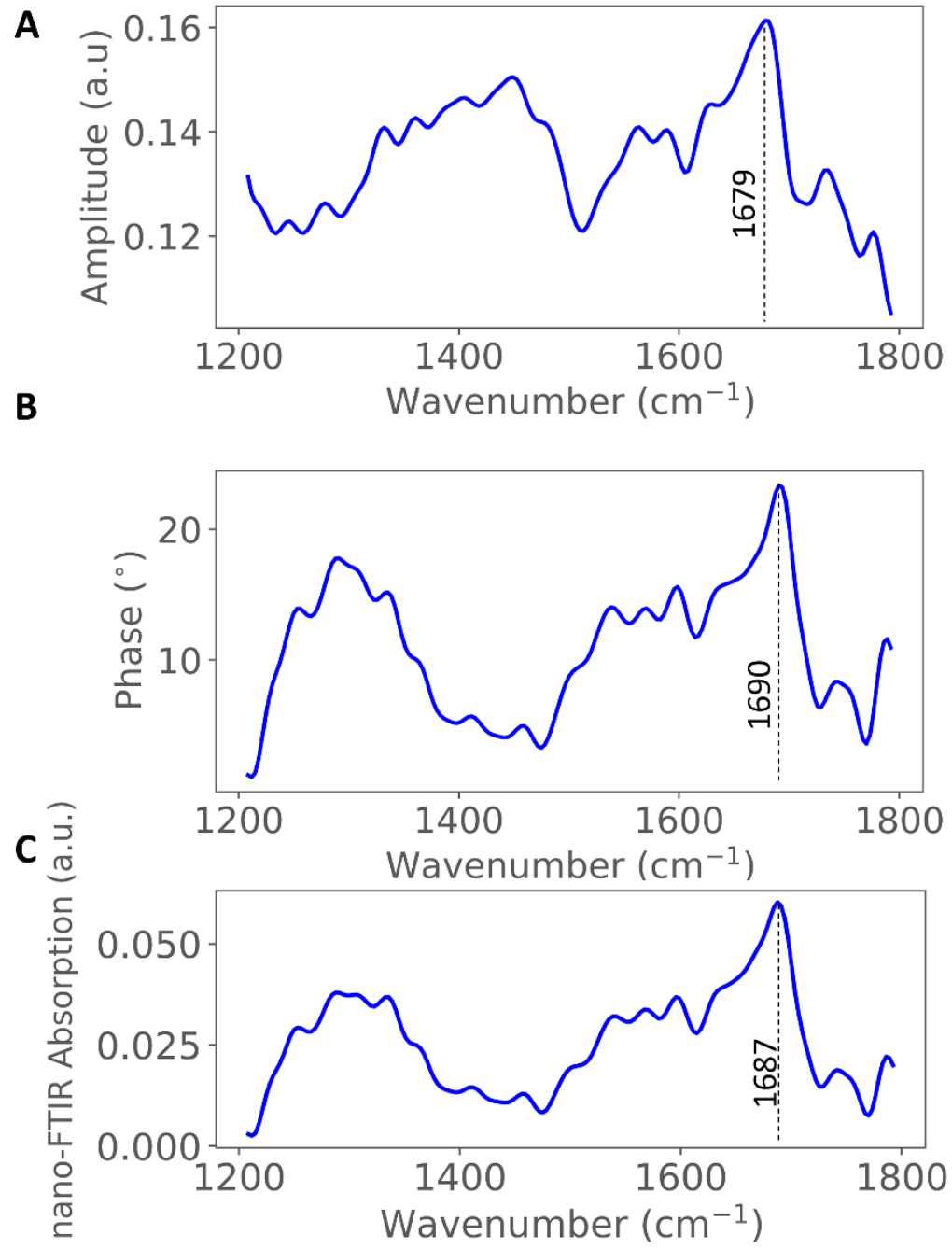

Figure S6. Nano-IR spectra of cell wall layers of sorghum fresh leaf sclerenchyma. A) Amplitude spectrum. B) Phase spectrum. C) Calculated nano-FTIR absorption spectrum showing the strongest spectral feature at $1687 \mathrm{~cm}^{-1}$ assigned to $\mathrm{C}=\mathrm{O}$ vibration that could indicate the presence of proteins or aromatic compounds (lignin). 